\title{
Research and application of the flood simulation and operation model in Beijing
}

\author{
Fuxin Chai ${ }^{1, *}$, Shu Liu ${ }^{1}$, Yang Sun ${ }^{2}$, and Fenglin $\mathrm{Huo}^{2}$ \\ ${ }^{1}$ State Key Laboratory of Simulation and Regulation of Water Cycles in River Basin, China Institute of Water Resources and \\ Hydropower Research, Beijing 100038, China \\ ${ }^{2}$ Beijing water emergency center, Beijing 100038, China
}

\begin{abstract}
This study built a coupling model of hydrology and hydrodynamics for flood forecasting and reservoir-dam joint scheduling. And using HTML5, java, and WebGIS technology, developed a flood control operation system based on $\mathrm{B} / \mathrm{S}$ framework for Beijing, integrated real-time information and fundamental information of metrology, rainfall, water flow, engineering situation, and waterlogging of Beijing. Through verification, the system has been successfully adopted to analyze the 2018 Ampil typhoon and provide a scientific basis for reservoirs operation including Yaoqiaoyu, Shachang, Haizi, ensuring safety of the reservoirs and reducing the economic loss of downstream area of reservoir.
\end{abstract}

\section{Introduction}

Due to influence of global climate changes, extreme weather and increasing urbanization, Beijing has seen more and more floods, which inflicted social economic losses. From 1991 to 2016, seven floods and 13 waterlogging disasters have wreaked havoc in Beijing. In particular, the storm flood in July 21st 2012 caused considerable economic and social losses in south-eastern Beijing. Flood scheduling systems provide effective support for flood-control policy-making and technical support to reduce harm and losses of flood disasters [15]. Therefore, the Water Supplies Bureau of Beijing began to build flood control operation system for major river basins in Beijing, constructed a flood simulation and operation system for Beijing, which was used during the flood season in 2018. The simulation system integrated the metrological, rainfall, water flow, engineering, and waterlogging information in Beijing, built a hydrology and hydrodynamics coupling flood forecast and a reservoir-dam operation model; by using Java programming language and database building technologies, a flood control operation system based on $\mathrm{B} / \mathrm{S}$ framework for Beijing was built to realize functions including flood situation analysis, flood control operation, operation result simulation, operation result assessment and approval. Meanwhile, the HTML5 technology is used to develop mobile apps, realize flood information searching, mobile inspection and flood situation reporting and other functions, providing integrated flood management decision-making support for Beijing.

\section{Flood simulation and scheduling model}

The flood simulation and scheduling model consists of multiple modules - a rainfall runoff model, a surface convergence model, a river convergence model, a reservoir dispatching model, a sluice gate and rubber dam application model. The structure of the model is shown in Figure 1.

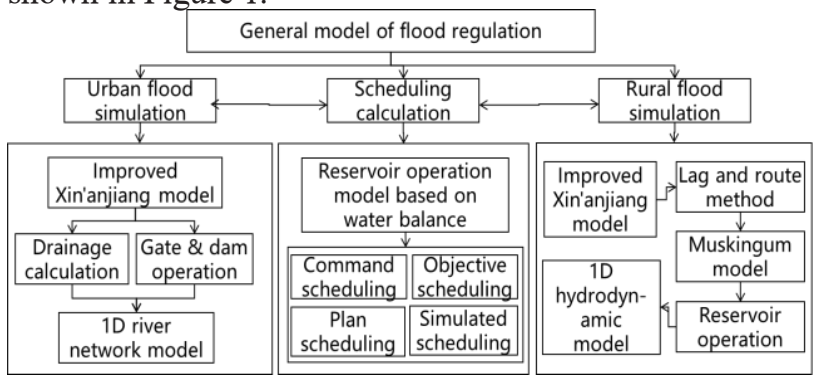

Fig. 1. Structure of the flood scheduling model

\subsection{Runoff model}

Based on the Xin'anjiang model of the sources of three rivers, the vertical mixed runoff model was adopted to combine the water storage capacity area distribution curve and infiltration capacity distribution curve of the watershed vertically. The vertical combination of full runoff and super seepage runoff makes the runoff model universal, suitable for humid, arid, and semi-arid and semi-humid areas, especially for the hilly, urban, and non-urban plains of Beijing has the same effect.

Based on the terrain and river characteristics, Beijing was divided into 172 sub-basins, and based on 121

\footnotetext{
*Corresponding author: chaifuxin@163.com
} 
rainfall monitoring stations, the surface rainfall was calculated by using the Thiessen polygons, and then the runoff model was used to calculate the rainfall runoff.

\subsection{Basin/drainage area confluence calculation}

A lag computing model was used to compute the basin confluence. According to the definition of a linear hydrological system, the transition and regulation of flood waves in the course of channel propagation can be regarded as floods flowing through $\mathrm{n}$ serial "linear channels" and n serial "linear reservoirs". The former features only transition and no regulation, while the latter flattens the waves. The lag computation model was used for computation of confluence of small watersheds and urban drainage areas.

\subsection{River flood evolution model}

According to river data and computation requirements for urban and non-urban areas, a one-dimensional hydrodynamic model was used in urban areas, and the Muskingum models were used in hilly areas and nonurban areas to compute river confluence.

The one-dimensional hydrodynamic model is discretized using the explicit finite difference method, which can deal with the dry bed problem well, and it is convenient to add various water conservancy projects. The integration form of the LAX format was adopted to arrange the water level in the middle of the flow section for the flow and water level variables. After obtaining the flow through the difference method, the continuous equation is processed by the finite volume method to solve the water level.

Beijing has constructed 20 Muskingum and river flood evolution models. At the same time, it built a onedimensional river network hydrodynamic model for rivers with a total length of $463.48 \mathrm{~km}$ and 1947 sections including major rivers such as the Chaobai River, the North Canal, and the Yongding River.

\subsection{Dam gate scheduling model}

Dam gate scheduling is divided into two types: large and medium reservoir scheduling and urban dam dispatching. Large and medium-sized reservoirs are based on water balance equations and use the water level-storage, water level-discharge relationship curves for scheduling computation. Scheduling methods include pre-planned scheduling, simulated scheduling (discharge-controlled scheduling and gate-based scheduling), and target scheduling. The dam gate scheduling in the urban area adopts the weir gate formula, which is calculated according to the upstream and downstream water levels and the gate opening degree. There are three types of scheduling methods - plan scheduling, given gate opening degree scheduling, and leak control scheduling.

A total of 20 large and medium-sized reservoirs and 82 sluice gates in the urban area were constructed.

\subsection{Model coupling}

Flood scheduling in major river basins in Beijing involves multiple models such as runoff generation model, river network evolution model, and scheduling analysis model, especially the coupling of the runoff generation hydrological model and the one-dimensional hydrodynamic river network model is even more critical. This time, these models are coupled from two aspects of time and space.

Spatially, through the refinement of urban drainage/catchment zoning, coupling the runoff and slope junction nodes with the one-dimensional hydrodynamic section of the river.

In terms of time, the computation period of the hydrological model is generally at the hour level, and the hydrodynamic model is at the second or the minute level. When the hydrological model computation results are docked with the hydrodynamic model, linear interpolation is performed. The whole hour of data is considered when the models are docked.

\section{Model verification}

Using the constructed model and system, the model verification and parameter calibration of two typical rainstorms in Beijing in recent years were performed to verify the reliability of the model. The simulation results of the rainstorm are shown in Figure 2 and Figure 3. The Figures show that the integrated model can meet the needs of flood prevention management.

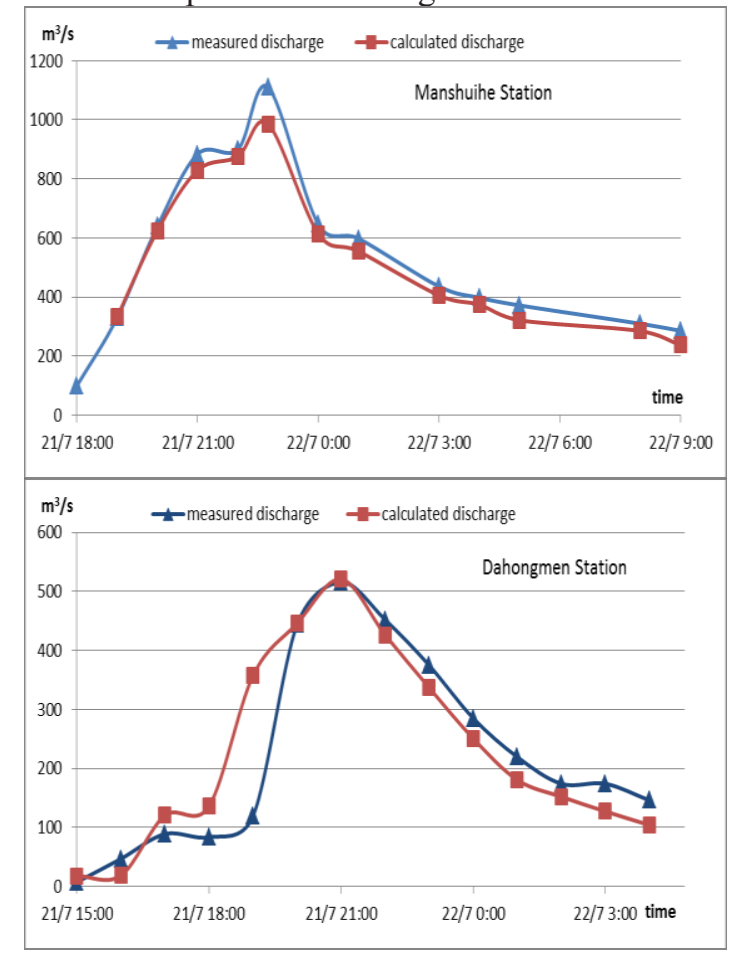

Fig. 2. Verification result for the "7.12" rainstorm in 2012 


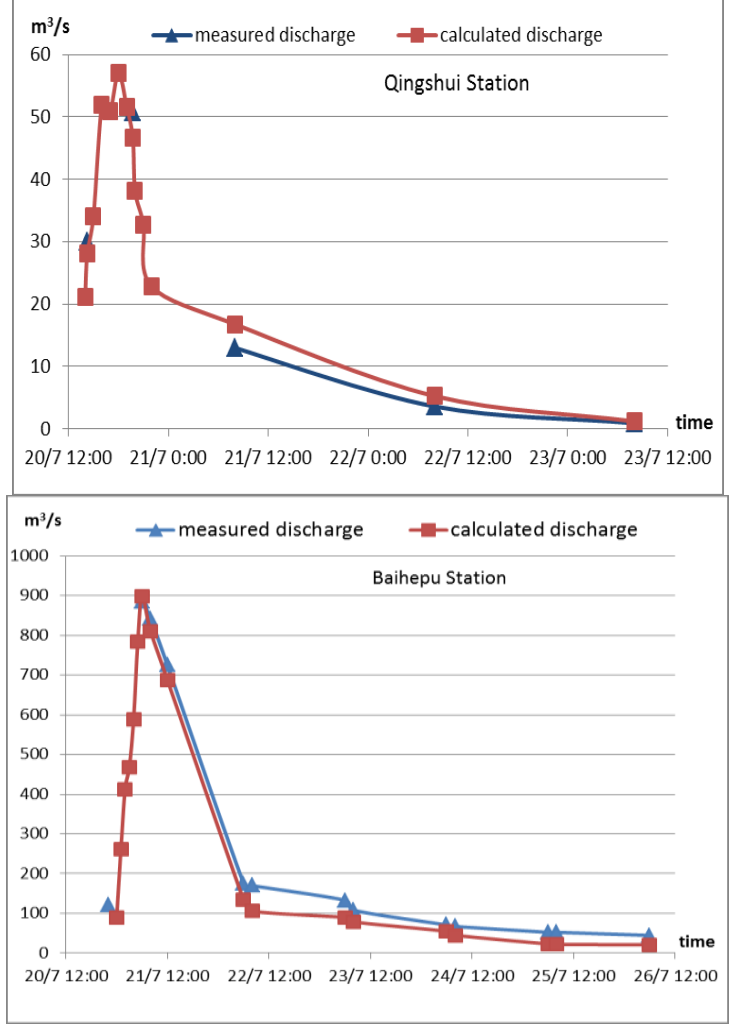

Fig. 3. Verification result for the " 7.20 " rainstorm in 2016

\section{Model application}

\subsection{Application overview}

In July 2018, affected by the Ampil typhoon, from 00:00 on the 24th to $18: 00$ on the 24th, Beijing's 18-hour accumulated surface rainfall was $37.27 \mathrm{~mm}$, of which the northern North Canal and the Ji Canal received a larger amount of rainfall, and the accumulated surface rainfall in the Ji Canal Basin was $93.23 \mathrm{~mm}$. The largest point rainfall occurred in Haizi Reservoir, where the accumulated rainfall for 18 hours was $124.9 \mathrm{~mm}$, reaching a 5-year record high, and the maximum rainfall for 1 hour was $24.8 \mathrm{~mm}$. The water of three large and medium-sized reservoirs rose rapidly, among which the maximum water level in Haizi Reservoir was $2.29 \mathrm{~m}$, and the maximum water level in Xiqiao Reservoir was $1.36 \mathrm{~m}$. The three reservoirs had a direct impact on the flood prevention safety of the reservoir and its downstream channels and even Pinggu District. Utilizing the Beijing flood simulation and scheduling model and system constructed this time, under the premise of rainfall and hydrological forecasting of the Beijing Meteorological Bureau and the Hydrological Station, scheduling and analysis were carried out to provide techniques for flood prevention consultation and emergency decision-making.

\subsection{Flood forecast and engineering scheduling}

According to the early warning information released by the Beijing Meteorological Observatory and the 24-hour precipitation forecast results of the Central Meteorological Observatory, it was speculated that the accumulated rainfall in the Jiyun River Basin might reach $100 \mathrm{~mm}$, the maximum 1-hour rainfall might reach $33.3 \mathrm{~mm}$, and the regional 1-hour maximum rainfall might reach $40-60 \mathrm{~mm}$. The main rainfall process occurred from 16:00 on 23rd July to 16:00 on 24th July. According to the results of meteorological forecast, rapid forecast and scheduling was carried out. According to the forecast of future rainfall, after analysis and computation, the two reservoirs exceeded the limit levels of floods, namely Xiqiao Reservoir and Haizi Reservoir.

1) The highest water level of Xiqiao Reservoir was $213.93 \mathrm{~m}$, which occurred at 17:00 on 24th July 2018, and the over-flood limit water level was $0.43 \mathrm{~m}$.

2) The highest water level of Haizi Reservoir was $109.66 \mathrm{~m}$, appearing time at 23:00 on 24th July, 2018, and the limit water level for over flooding is $1.16 \mathrm{~m}$.

According to the scheduling plan, the Haizi Reservoir and Huangsongyu Reservoir would not discharge floods under this scheme, and the Xiyu Reservoir would release $100 \mathrm{~m} 3 / \mathrm{s}$. The reservoir scheduling results are shown in Table 1.

Table 3. Reservoir scheduling results of Jiyun River Basin (100 mm rainfall)

\begin{tabular}{|c|c|c|c|c|}
\hline Reservoir & $\begin{array}{c}\text { Max } \\
\text { inflow } \\
\left(\mathbf{m}^{\mathbf{3}} / \mathbf{s}\right)\end{array}$ & $\begin{array}{c}\text { Highest } \\
\text { level } \\
(\mathbf{m})\end{array}$ & $\begin{array}{c}\text { Max } \\
\text { outflow } \\
\left(\mathbf{m}^{\mathbf{3}} \mathbf{s} \mathbf{s}\right)\end{array}$ & $\begin{array}{c}\text { Rate of peak } \\
\text { cutting } \\
(\mathbf{\%})\end{array}$ \\
\hline Haizi & 1007.53 & 109.66 & 0 & 100.00 \\
\hline Huangsongyu & 118.4 & 202.21 & 0 & 100.00 \\
\hline Xiyu & 192.58 & 213.93 & 100 & 48.07 \\
\hline
\end{tabular}

Due to the more accurate forecast of the total rainfall in the Jiyun River Basin, the $100 \mathrm{~mm}$ forecast was $7.26 \%$ larger than the actual surface rainfall of $93.23 \mathrm{~mm}$. The predicted maximum water level of Haizi Reservoir was $0.65 \mathrm{~m}$ lower than the actual maximum water level, which was mainly due to the occurrence of double peaks due to the impact of frontal rain in the later period of the typhoon. The predicted maximum water level of the reservoir (at 23 O'clock on 24th July) was measured at $109.67 \mathrm{~m}$ (consistent with the forecast value was 109.66 $\mathrm{m})$. The highest water level of Huangsongyu Reservoir was $202.21 \mathrm{~m}$, which was close to the forecast value. The highest water level of Xisong Reservoir was $214.28 \mathrm{~m}$, which was $0.35 \mathrm{~m}$ higher than the scheduling result. After the backflow into the reservoir flood peak, the maximum reservoir flood peak was in line with the actual situation. The flood peaks were also very close, so flood forecasting and scheduling were generally more accurate.

Affected by the uncertainty of typhoon rainfall and the uncertainty of flood scheduling in the downstream river, the pre-discharge operation ensured the safety of the upstream and downstream rivers of the reservoir. Therefore, the forecast and scheduling of the three reservoirs in the Jiyun River Basin were timely and successful. 


\section{Conclusions}

According to the characteristics of flood disasters in Beijing, a flood simulation and operation model based on coupling of the runoff model, the confluence model, the one-dimensional river network model, and the reservoir and gate operation model were constructed. Based on the existing software and hardware environment in Beijing, by integrating various data, a flood forecast and operation system was developed. The model verification and application show that the computation accuracy of the model and system meets flood control requirements, ensuring the safety of the reservoir and minimizes the socio-economic losses in the downstream areas along the river banks, providing strong support for flood control decision-making and command in Beijing.

\section{Acknowledgement}

The authors thank the support of the projects of the 13th Five-year Plan of National Scientific Research and Development (No. 2016YFC0803107).

\section{References}

1. S.L. Guo, Hui Peng, J.X. Wang et.cl. Design and development of reservoir operation system. Journal of China Hydrology. 14, 3:4-7 (2001)

2. C.T. Cheng, C.P. OU, B.D. Wang. Integrated management technology for flood control system of reservoirs. Journal of Hydraulic Engineering, 32, 3:77-81 (2001)

3. G.L. Wang, Yong Peng, Bin He et.cl. Study of B/S \& C/S-based flood control decision-making support system and its application. Journal of Dalian University of Technology, 50, 2: 258-263 (2010)

4. S.Y. Hu, D.D. Song, Y.X. Wu et.cl. Framework and Design of the Yangtze River Flood Control Decision Support System. Advances in water science, 7, 4 :415 (1996)

5. Daniel Che, Larry W. Mays. Application of an Optimization/Simulation Model for Real-Time Flood-Control Operation of River-Reservoirs Systems. Water Resources Management, 31,7: 2285-2297 (2017) 